\title{
Immersion Scatterometry for Improved Feature Resolution and High Speed Acquisition of Resist Profiles
}

\author{
Fred L. Terry, Jr. (Dept. of EECS, University of Michigan, Ann Arbor, MI 48109-2122) and \\ Joseph J. Bendik ( Dynamic Intelligence, Inc. (DI ${ }^{2}$ ), Escondido, CA 92025)
}

\begin{abstract}
Specular-mode spectroscopic scatterometry is currently being used as an in-line metrology tool for wafer-to-wafer process monitoring and control in lithography and etch processes. Experimental real-time, in situ demonstrations of critical dimension monitoring and control have been made for reactive ion etching. There have been no similar demonstrations of real-time control in the critical step of resist development. In this paper, we will show the results of a simulation study on the use of scatterometry in an immersion mode both to improve resolution and to act as a real-time monitor for photoresist topography evolution during development. We have performed realistic simulations of the experimental performance by using Prolith ${ }^{\mathrm{TM}}$ to generate developing resist profiles vs. time and a rigorous couple wave algorithm (RCWA) simulator (modified to include the immersion ambient) to generate simulated scatterometry data. We have examined several modes of operation of the proposed measurement including specular and $1^{\text {st }}$ order scattered modes using spectroscopic ellipsometry and reflectometry. For our simulations, we have used pure water to approximate the developer refractive index. We have created realistic simulation data by adding appropriate amounts of random noise to perfect simulations, and then used regression analysis to extract profiles from these data. Water immersion increases feature shape resolution for small period gratings by increasing the scattering into real diffracted modes.
\end{abstract}

\section{Introduction}

Specular-mode spectroscopic ellipsometry (SE) or reflectometry (SR) measurements from periodic gratings ("scatterometry", or optical critical dimension metrology-OCD) have been successfully demonstrated as accurate methods for extracting detailed topography of integrated circuit structures and this method is rapidly becoming established as a important in-line metrology tool for manufacturing control. ${ }^{1,2,3,4,5,6,7}$ Integrated metrology variations have been demonstrated for wafer-to-wafer feedfoward and feedback control of photolithography and reactive ion etch steps. ${ }^{8,9,10}$ University research demonstrations have used both $\mathrm{SR}^{5,11}$ and $\mathrm{SE}^{12}$ for both ex situ, detailed topography extraction and in a real-time, in situ mode to monitor the evolution of topography in reactive ion etch systems during oxygen-plasma trim-back of photoresist lines, the evolution of shallow trenches in crystalline $\mathrm{Si}$, and the etching of poly-Si gate test structures.

These prior efforts all used either air or vacuum as the ambient. In this paper, we report on a simulation study of immersion mode scatterometry for both in-line and in situ applications. We find that just as in the case of immersion lithography, immersion scatterometry has the strong potential to improve the resolution limitations of air-ambient scatterometry. Also, water immersion would allow the measurement beam to be focused into a smaller spot, and thus would aid in reducing the required scatterometry test area size.

For in situ applications, the use of a very high speed array detector-based instrumentation scheme (either in spectroscopic ellipsometry or spectroscopic reflectometry modes) could allow the real-time monitoring of photoresist profile evolution during development. The practicality of this for real-time process control is not completely obvious; however, specialized development test cells would allow very accurate real-time monitoring of the development process. This would enable the more rapid creation of accurate develop-curve models. We will refer to this notion as high speed acquisition of resist profiles (HARP). 


\section{Modeling}

Our immersion mode scatterometry modeling codes were written in MatLab ${ }^{\mathrm{TM}}$ and made use of the wellknown methods of Moharam, et al. ${ }^{13}$ with the convergence-aiding corrections for the TM case by Li. ${ }^{14}$ The formulation of Moharam easily allows modeling of the immersion mode case by including the refractive index behavior of the ambient (the incident media or ambient term $n_{I}$ in equation 3 of reference 13). While $n_{I}=1$ for air or vacuum mode scatterometry, it takes on larger values for liquid immersion cases. For all of the simulations in this paper, we assumed that the immersion ambient was pure $\mathrm{H}_{2} \mathrm{O} .{ }^{15}$ This ambient currently seems the best for immersion scatterometry for exactly the same reasons it is attractive for immersion lithography - relatively high refractive index and good UV transmission. For our photolithography development simulations, it would have been desirable to use the refractive index functions of the actual developers; however, these are not currently available so water was used as an approximation to these aqueous solutions. We will make comments later in this paper about possible measurement solutions to this problem and on the possible limitations on in situ development monitoring.

Prolith $^{\mathrm{TM}}$ (version 8.12 ) grating simulations for $\sim 100 \mathrm{~nm}$ linewidth grating structures were derived from an AZ/Clariant AX1020P 193nm chemically amplified resist (CAR) system that was originally calibrated for 130nm lines $(325 \mathrm{~nm}$ pitch). Fine structure in the development contours is realized by under-developing the resist profile and extracting the development contours with a time step $<.25$ seconds. The following table highlights the important simulation parameters used in this study.

\begin{tabular}{|l|}
\hline $\mathrm{NA}=0.85$ \\
\hline Sigma $=0.65$ \\
\hline $90 \mathrm{~nm}$ lines $/ 270 \mathrm{~nm}$ pitch $:$ binary \\
\hline CAR AX1020P \\
\hline $400 \mathrm{~nm}$ resist $/ 44 \mathrm{~nm}$ AR $2 /$ Poly Silicon \\
\hline $\mathrm{PAB}, \mathrm{PEB}: 110 \mathrm{C} / 60$ seconds \\
\hline $\mathrm{SF}=1, \mathrm{Vector}$ \\
\hline $\mathrm{With} /$ without coma $(5 \mathrm{mwaves})$ \\
\hline $\mathrm{F}=-.05 \mathrm{~nm}, \mathrm{E}=20 \mathrm{mj} / \mathrm{cm}^{2}$ \\
\hline Develop times $(.25-60$ seconds $)$ for contour detail \\
\hline
\end{tabular}

Table 1 Prolith parameters for resist grating structures

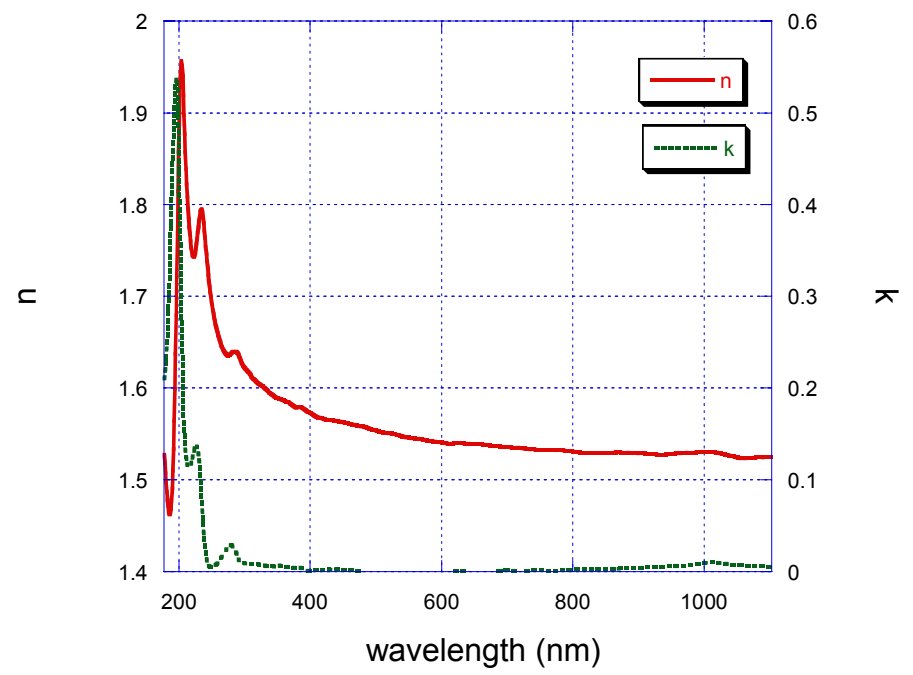

Figure 1 Refractive indices of photoresist used in this study. 
A variety of different size features were examined in the course of this study, but in this paper we will concentrate on simulations $\sim 100 \mathrm{~nm}$ lines and on scaled reductions from these simulations to examine features down to $\sim 30 \mathrm{~nm}$. The refractive index of the simulated resist is shown in Figure 1.

\section{Resolution Improvements with Immersion Scatterometry}

Scatterometry is capable of some level of critical dimension and feature shape extraction even in cases of extremely small linewidth/short period grating structures, but resolution of fine detail is reduced at smaller dimensions. ${ }^{16}$ This situation can be somewhat improved by water immersion. It is our hypothesis that coupling energy into real diffracted modes will increase the sensitivity of specular mode scatterometry since the efficiency and phase of this scattering will be sensitive to the feature shape.

Significant benefits can be obtained in cases of short period gratings. When the grating period becomes sufficiently small compared to the minimum free space wavelengths used in the measurement, then it is easily shown that the only real diffracted mode (for reflection) which occurs is the $1^{\text {st }}$ order backscattered mode $(m=-1)$. Starting with the grating equation:

$$
\sin \theta_{D}=\sin \theta_{i}+\frac{m \lambda_{o}}{\Lambda n_{I}}
$$

(where $\Lambda$ is the grating period, $\lambda_{\mathrm{o}}$ is the free space wavelength, $\mathrm{n}_{\mathrm{I}}$ is the ambient refractive index, and $\theta_{\mathrm{i}}$ and $\theta_{\mathrm{D}}$ are the angles of incidence and diffraction) we can readily see that for small period gratings there will be a real (non-

evanescent) reflection diffraction mode only if $\sin \theta_{D}>-1\left(\left|\theta_{D}\right|<90^{\circ}\right)$ and thus:

$$
\lambda_{o}<\Lambda n_{I}\left(1+\sin \theta_{i}\right)
$$

Clearly, larger values of $\sin \theta_{i}$ (large angles of incidence) will improve this situation. For the commonly employed ellipsometry angle of $75^{\circ}, \sin \theta_{i} \approx 0.966$. We will use this condition for the simulations in this paper. To illustrate the strong effects of water immersion on scatterometry data, we simulated a $\sim 40 \mathrm{~nm}$ photoresist line structure in a grating of with $\Lambda=90 \mathrm{~nm}$. We assumed a photoresist directly on a Si substrate. The lineshape is a 3x scaled reduction of the Prolith simulation discussed later and is shown in Figure 2 below. In air, the $1^{\text {st }}$ real diffracted modes occur for $\lambda_{0}<176.9 \mathrm{~nm}$. This range would be accessible for purged or vacuum UV ellipsometers, but not for conventional systems. In water immersion, at $U V$ wavelengths $n_{1} \sim 1.39$, yielding a similar requirement of $\lambda_{0}<273.2 \mathrm{~nm}$ (well within the conventional air accessible UV range). Ideal simulations of air mode and immersion mode spectroscopic ellipsometry and spectroscopic reflectometry measurements are shown in Figure 3 and Figure 4. These simulations show much stronger structure in both the ellipsometry and reflectometry data in the water immersion cases in the region were this $m=-1$ diffraction mode becomes possible. 

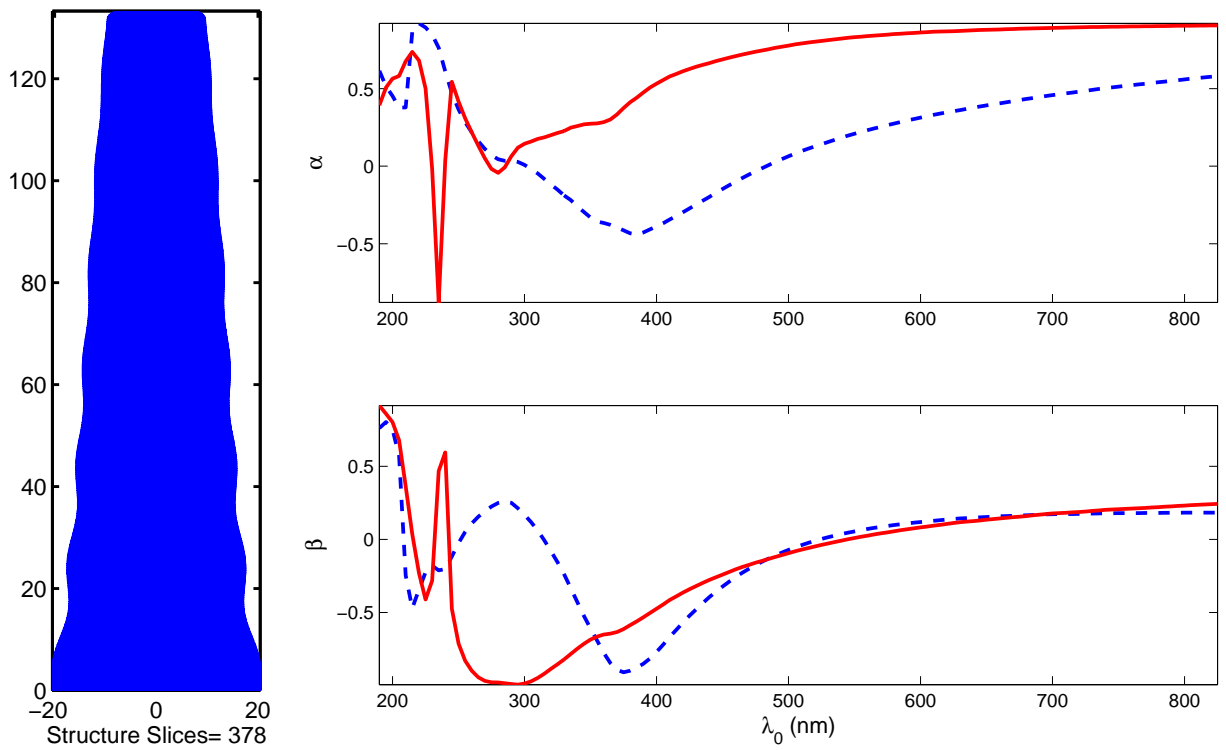

Figure 2 (left) Photoresist lineshape for 90nm period grating simulations.

Figure 3 (right) Spectroscopic ellipsometry simulations at $75^{\circ}$ in air (dashed lines) and $\mathrm{H}_{2} \mathrm{O}$ (solid lines) for the $90 \mathrm{~nm}$ period photoresist structure. Note the strong structure in the immersion mode data below $300 \mathrm{~nm}$.
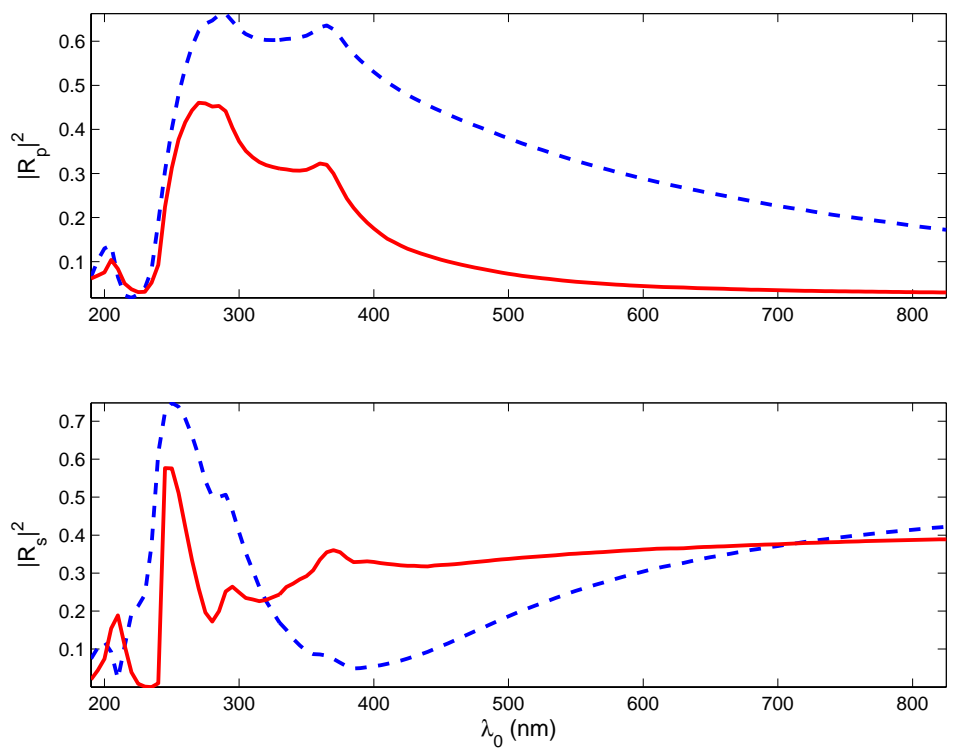

Figure 4 Spectroscopic reflectometry simulations at $75^{\circ}$ in air (dashed lines) and $\mathrm{H}_{2} \mathrm{O}$ (solid lines) for the $90 \mathrm{~nm}$ period photoresist structure. Note the strong structure in the s-mode reflectance.

This increase in diffraction-related structure in the data improves the sensitivity of the measurement to feature shape. To quantitatively illustrate this, we took both ideal simulations (air \& $\mathrm{H}_{2} \mathrm{O}$ immersion) and added Gaussian random noise to the data (both the $\alpha \& \beta$ parameters) with a standard deviation $\sigma=0.02$. Then we used these data sets as data for regression extraction of the feature shape (just as we would attempt to do in a physically real experiment). Since we know the standard deviation of the noise and know that it is purely random (no systematic errors), we can use the 
well known reduced chi-squared error formulation to represent the mean square error. ${ }^{17}$ The application of this error formulation has been applied extensively to spectroscopic ellipsometry data modeling by Jellison. ${ }^{18}$ When $\chi \sim 1$, we can say that the model well-represents the data and that little additional information can be obtained (we have fit the result to within the noise limits). If $\chi>>1$, then there is more information in the data than is being extracted by the model and there is danger of poor conclusions due to the bad model. If $\chi<1$, then we have over-fit the data and some or all of our extracted parameters may have no physical significance. While these are not prefect statistical tests, they are much better than arbitrary goodness of fit parameters.

Using this simulated data, we fit a curved trapezoid model (a quadratic sidewall shape) and included the possibility of blanket undeveloped bottom resist layer. For each fitted curved trapezoid segment $j$, the width of segment at a given point y measured down from the top of the segment is given by:

$$
w(y)=\left[m_{j 0}+m_{j 1}\left(\frac{y}{h_{j}}\right)+m_{j 2}\left(\frac{y}{h_{j}}\right)^{2}\right] \Lambda
$$

where the m's are the sidewall polynomial shape parameters and $h_{j}$ is the segment total height. The approach can be obviously generalized to higher order polynomials. The results of this fit are shown in Figure 5 for both the air and water immersion cases. The fitted parameters are summarized in Table 2.

Remarkably, these results show that air-mode scatterometry is not too bad for feature shape extraction even at these small dimensions. However, some obvious errors can been seen readily in the overlay of the air-fit topography result with the actual lineshape. In particular, the height of the main resist line was underestimated, while the blanket undeveloped resist layer (which should have been zero) was significantly overestimated. Furthermore, the low $\chi$ value suggests that significant further information cannot be extracted from this data. For the water immersion case, we can see that the visual quality of the fitted shape vs. actual lineshape is better. Further, from the table we can see that the confidence limits of the fitted parameters are significantly better. We also examined the cross-correlation matrices for these fits (not shown due to space limitations), and the immersion case shows less heavily correlated terms. All of this is consistent with the additional structure in the data of the immersion case due to the diffraction mode. We expect that this trend would become even more pronounced if the noise in the data were larger.
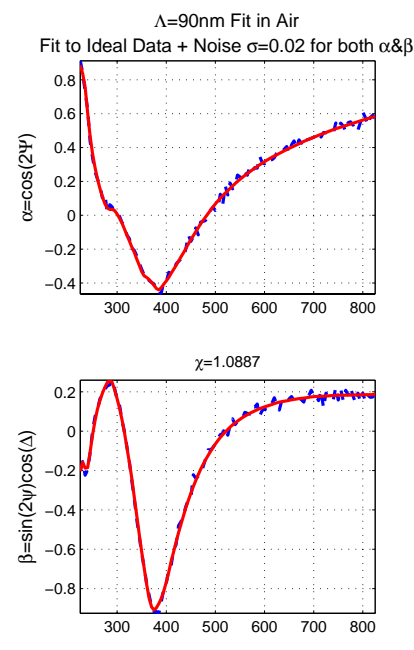
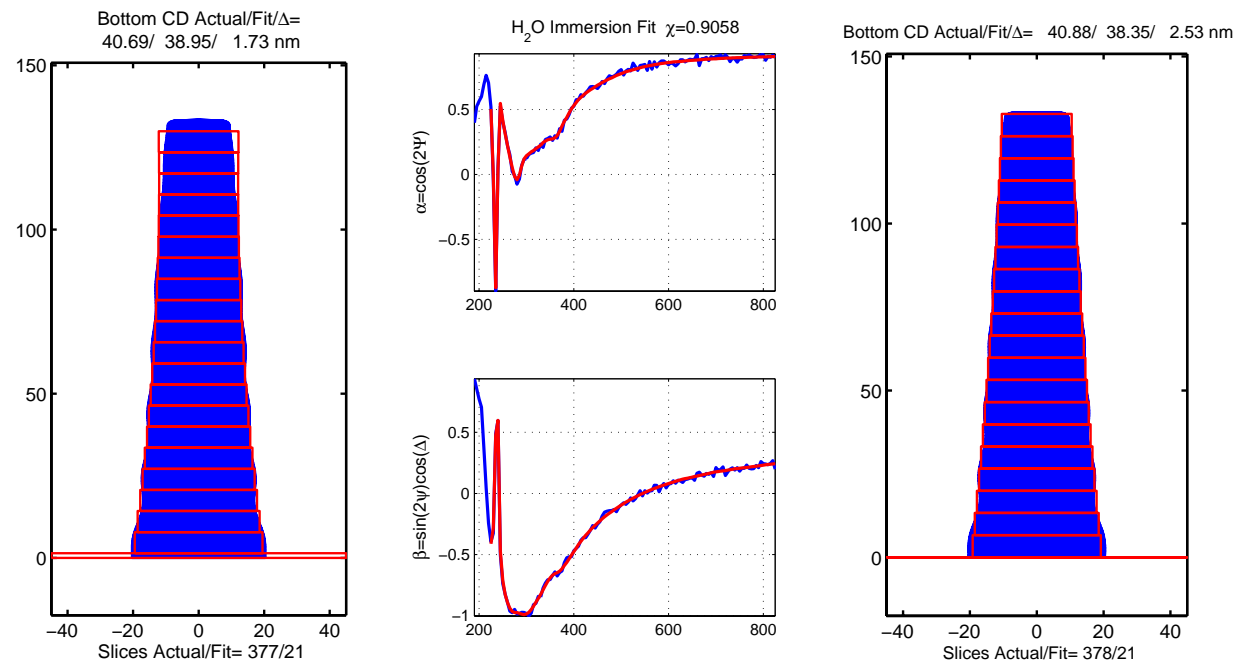

Figure 5 Fits to $90 \mathrm{~nm}$ for data taken in air (left) and the data taken in $\mathrm{H}_{2} \mathrm{O}$ (right). For both figures, random noise with a $\sigma=0.02$ was added to the ideal simulation of $\alpha \& \beta$. Low $\chi$ 's were obtained for both. However, the air result shows a obvious height error and the $\chi \sim 1.09$ value shows that basically no more can be obtained from this data. The fit of the water immersion case is clearly better. The chi of 0.9058 indicates we have pushed this shape data close to the limit (and perhaps over). 


\begin{tabular}{|l|c|c|c|c|}
\hline \multicolumn{1}{|c|}{ Parameter } & $\begin{array}{c}\mathrm{H}_{2} \mathrm{O} \\
\text { Immersion } \\
\text { value }\end{array}$ & $\begin{array}{c}\mathrm{H}_{2} \mathrm{O} \\
\text { Immersion } \\
68.30 \% \\
\text { Conf. } \\
\text { Limit }\end{array}$ & $\begin{array}{c}\text { Air } \\
\text { value }\end{array}$ & $\begin{array}{c}\text { Air } \\
68.30 \% \\
\text { Conf. } \\
\text { Limit }\end{array}$ \\
\hline Seg 1 Height $(\mathrm{nm})$ & 132.705 & 0.549 & 128.529 & 1.52226 \\
\hline Seg 1 Top Width $(\mathrm{nm})$ & 20.691 & 0.1 .229 & 24.4053 & 2.36763 \\
\hline Seg 1 Slope & 0.1059 & 0.0735 & 0.05447 & 0.11919 \\
\hline Seg 1 Curvature & 0.0979 & 0.0817 & 0.22588 & 0.116975 \\
\hline Seg 2 Height $(\mathrm{nm})$ & 0.09 & 0.8775 & 1.41399 & 1.06542 \\
\hline
\end{tabular}

Table 2 Fit parameters for both the air and water immersion results of Figure 5. The actual simulated structure height (segment 1 height) was $133.33 \mathrm{~nm}$ and the actual bottom resist height (segment 2 height) was 0 .

\section{Simulations of 100nm Line Developing}

To simulate real-time monitoring of resist profile evolution, we use the Prolith ${ }^{\mathrm{TM}}$ simulations of the $\sim 100 \mathrm{~nm}$ lines described earlier. In our conference presentation, high accuracy animations (films) of the development process will be presented and will be posted on one of the author's websites following the conference. ${ }^{19}$ For all the simulations shown in this section, we used a $\Lambda=270 \mathrm{~nm}$ grating structure, a resist thickness of $400 \mathrm{~nm}$, and a final resist line dimension of $\sim 100 \mathrm{~nm}$. Development times out to $20 \mathrm{~s}$ were simulated on a $0.1 \mathrm{~s}$ interval. Contour curves were fit to the Prolith $^{\mathrm{TM}}$ results to automatically create the RCWA slice structure. Depending on the complexity of the resist line shape between $\sim 150-390$ slices were used in the scatterometry simulations of the ideal data. As stated earlier, we assumed that the refractive indices of the developer were the same as $\mathrm{H}_{2} \mathrm{O}$. In this paper, we will present the results of fits to the data from two times during the development process. For the regression fit simulations, random noise $(\sigma=0.02)$ was added to the data in the same manner as described earlier for the small line simulations.
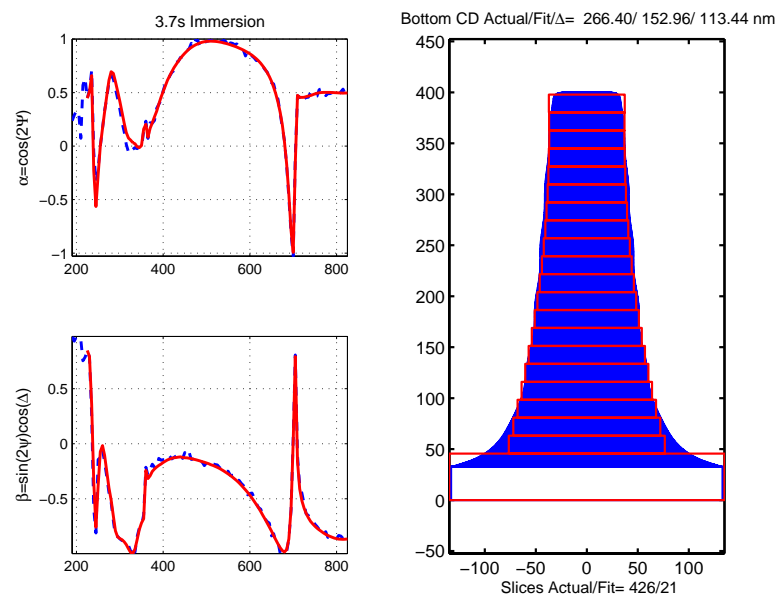

\begin{tabular}{|l|r|r|}
\hline Parameter & Value & $\begin{array}{c}68.3 \% \\
\text { Conf. } \\
\text { Limit }\end{array}$ \\
\hline $\begin{array}{l}\text { Seg 1 } \\
\text { Height (nm) }\end{array}$ & 352.188 & 1.2015 \\
\hline $\begin{array}{l}\text { Seg 1 } \\
\text { Top Width (nm) }\end{array}$ & 74.898 & 1.269 \\
\hline $\begin{array}{l}\text { Seg 1 } \\
\text { Slope }\end{array}$ & 0.0452 & 0.0047 \\
\hline $\begin{array}{l}\text { Seg 1 } \\
\text { Curvature }\end{array}$ & 0.0456 & 0.0226 \\
\hline $\begin{array}{l}\text { Seg 2 } \\
\text { Height (nm) }\end{array}$ & 45.603 & 0.7695 \\
\hline
\end{tabular}

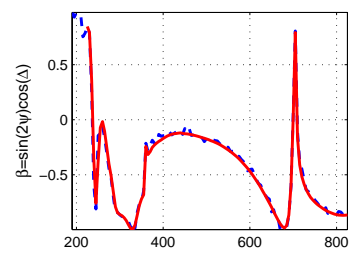

Figure 6 Simulated photoresist structure with 299 slices. SE scatterometry data simulated at $75^{\circ}$ AOI with random noise of 0.02 added to both $\alpha$ and $\beta$ (dashed curves). The regression fitted structure (trapezoid+curvature) had 20 slices. The reduced $\chi$ error $\sim 4.47$ indicates that while the fit is good, there is more information available from the data by improving the physical model. For this structure, including extra parameters to better describe the top curvature will improve the $\chi$ without over-fitting. 

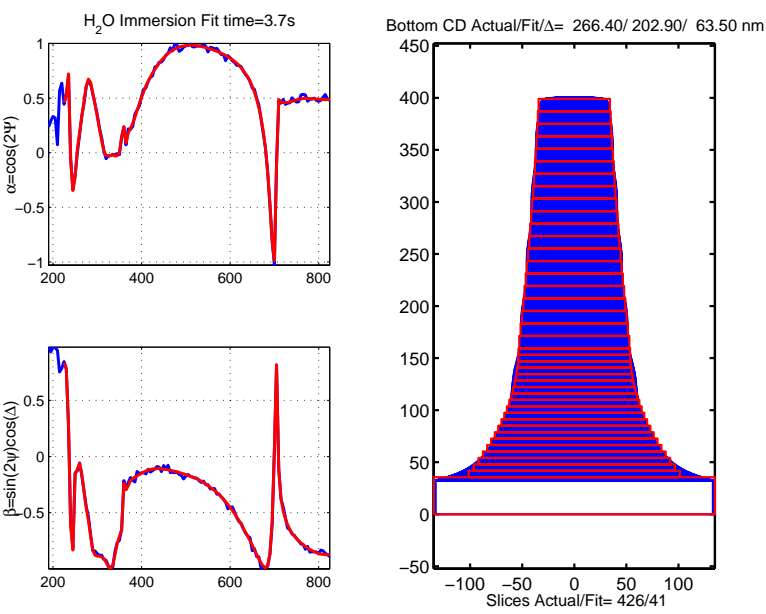

\begin{tabular}{|l|c|c|}
\hline Parameter & Value & $\begin{array}{c}68.3 \% \\
\text { Conf. Limit }\end{array}$ \\
\hline $\begin{array}{l}\text { Seg 1 } \\
\text { height (nm) }\end{array}$ & 239.652 & 36.153 \\
\hline $\begin{array}{l}\text { Seg 1 } \\
\text { Top Width (nm) }\end{array}$ & 67.959 & 1.863 \\
\hline $\begin{array}{l}\text { Seg 1 } \\
\text { Slope }\end{array}$ & 0.0879 & 0.04505 \\
\hline $\begin{array}{l}\text { Seg 1 } \\
\text { Curvature }\end{array}$ & 0.057 & 0.0202 \\
\hline $\begin{array}{l}\text { Seg 2 } \\
\text { height (nm) }\end{array}$ & 123.822 & 35.721 \\
\hline $\begin{array}{l}\text { Seg 2 } \\
\text { Slope }\end{array}$ & -0.0067 & 0.10665 \\
\hline $\begin{array}{l}\text { Seg 2 } \\
\text { Curvature }\end{array}$ & 0.3802 & 0.11185 \\
\hline $\begin{array}{l}\text { Seg 3 } \\
\text { height (nm) }\end{array}$ & 35.559 & 0.6075 \\
\hline
\end{tabular}

Figure 7 The same data as Figure 6 is fit with a 3 region model (top trapezoid+curvature, bottom trapezoid+curvature, bottom uniform resist layer). The fit $\chi$ is reduce to $\sim 0.944$ and all the fitted parameters are statistically significant (based on $68.3 \%$ confidence limits) except for the segment 2 slope (which is essentially 0 ). The cross correlation matrix does show strong coupling between the heights of the 2 curved trapezoid segments, but the correlated parameters trade-off to yield an overall accurate total height.

Figure 6 shows a resist profile and spectroscopic ellipsometry data curve at an intermediate point in the development process. The profile is not fully evolved and there is $\sim 200 \mathrm{~nm}$ of completely undeveloped resist remaining. An initial regression fit using a curved trapezoid on blanket resist model yields the results shown in this figure and accompanying table. The $\chi \sim 4.47$ suggests that more can be obtained from this data. Increasing the number of slices for the fit (from 20 as shown) does not appreciably change the error, indicating that it is an overall shape error rather than discretization error that is being detected by $\chi$. Increasing the model complexity to a curved-trapezoid on a curvedtrapezoid on blanket resist model yield the results shown in Figure 7 and a $\chi \sim 0.944$. As can be seen visually, the more complex curvature of the actual structure is better captured by this model and the $\chi$ and confidence terms indicate that this fit statistically valid.

As the feature evolves towards the fully developed case, the wall shape becomes more vertical and we return to the simpler curved-trapezoid on rectangle model. This is illustrated in Figure 8. Increasing the number of slices in the RCWA model from 20 (as shown in this figure) to 200 does not significantly change the $\chi$ error. The finer scale of the 200 slice fit does very slightly reduce the bottom CD error (by $\sim 1 \mathrm{~nm}$ ) vs. the 20 slice model. Even with water immersion, it appears that even data with small but still realistic values of noise would not allow unambiguous extraction of very fine shape detail such as the standing wave ripples in this data.

These results suggest that immersion mode scatterometry should be capable of yielding very valuable data for the rapid construction of resist development models. Two issues that will likely complicate the situation in actual implementation are: (1) the need for accurate information on the refractive indices of the developer solution; and . (2) the possibility that the developer solution will be absorbing in the UV region. The first problem may require a procedure for extraction of the refractive index behavior at the time of the specific experiment since there may be solution concentration variation effects. One possible method to address this would be the use of a standard blanket oxide wafer in the test cell prior to the resist development experiment. With an accurately known reference, extraction of the real part of the ambient solution index should be possible. If the absolute value of the reflectance was also measured, the imaginary part of the media index could also be extracted (assuming proper care is taken for window effects, reflection 
losses, etc.) If the imaginary part of the immersion media refractive index is too large, essentially all of the light will be absorbed and no data will be collected in that spectral region.
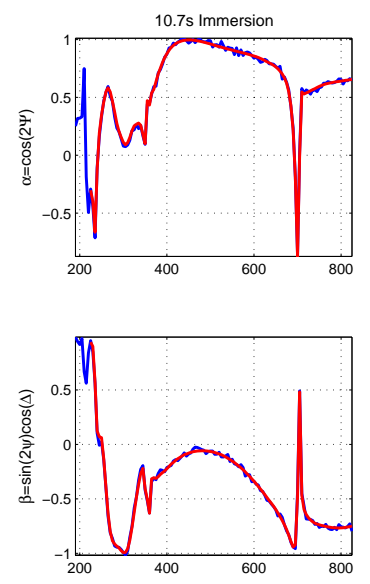

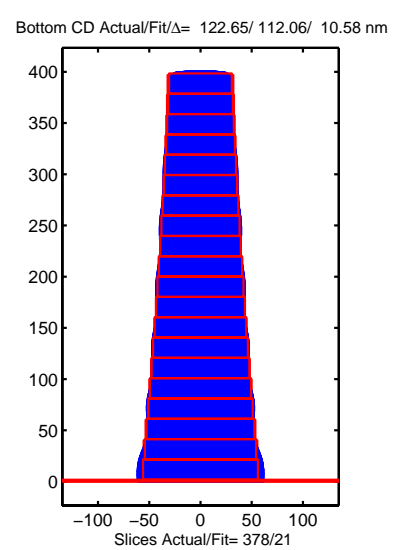

\begin{tabular}{|l|r|r|}
\hline Parameter & Value & \multicolumn{1}{|c|}{$\begin{array}{l}68.3 \% \\
\text { Conf. } \\
\text { Limit }\end{array}$} \\
\hline $\begin{array}{l}\text { Seg 1 } \\
\text { Height (nm) }\end{array}$ & 396.657 & 0.540 \\
\hline $\begin{array}{l}\text { Seg 1 } \\
\text { Top Width (nm) }\end{array}$ & 60.507 & 0.689 \\
\hline $\begin{array}{l}\text { Seg 1 } \\
\text { Slope }\end{array}$ & 0.131 & 0.012 \\
\hline $\begin{array}{l}\text { Seg 1 } \\
\text { Curvature }\end{array}$ & 0.067 & 0.012 \\
\hline $\begin{array}{l}\text { Seg 2 } \\
\text { Height (nm) }\end{array}$ & 1.701 & 0.405 \\
\hline
\end{tabular}

Figure 8 Simulated photoresist structure with 378 slices. SE scatterometry data simulated at $75^{\circ}$ AOI with random noise of 0.02 added to both $\alpha$ and $\beta$ (dashed curves). The regression fitted structure (trapezoid+curvature) had 20 slices. Increasing the number of fit slices to 200 has only negligible effect on the reduced $\chi$ error. The actual resist height was $400 \mathrm{~nm}$ (seg 1) with no bottom undeveloped resist (seg 2).

Absorption in the developer would limit the viable wavelength range of the scatterometry measurements. We attempted to simulate for this possibility by taking the data of Figure 8 and fitting the same model to the data over the more limited range of 400-800 $\mathrm{nm}$ (Figure 9). In this case, the $\chi$ drops a bit below 1 (0.9854), indicating that we have over-fit the data (here that our curvature parameter is probably no longer valid even though the confidence limit is showing an acceptable value). As is clear in the figure, even with this wavelength limitation there is sufficient information in the data to do useful extraction of critical dimensions and sidewall slopes. In this case, part of this sensitivity comes directly from the real diffraction mode ( $\mathrm{m}=-1$ again) effects on the specular data in the $\sim 700 \mathrm{~nm}$ range. For air mode measurements, these effects would be shifted up to $\sim 500 \mathrm{~nm}$. 

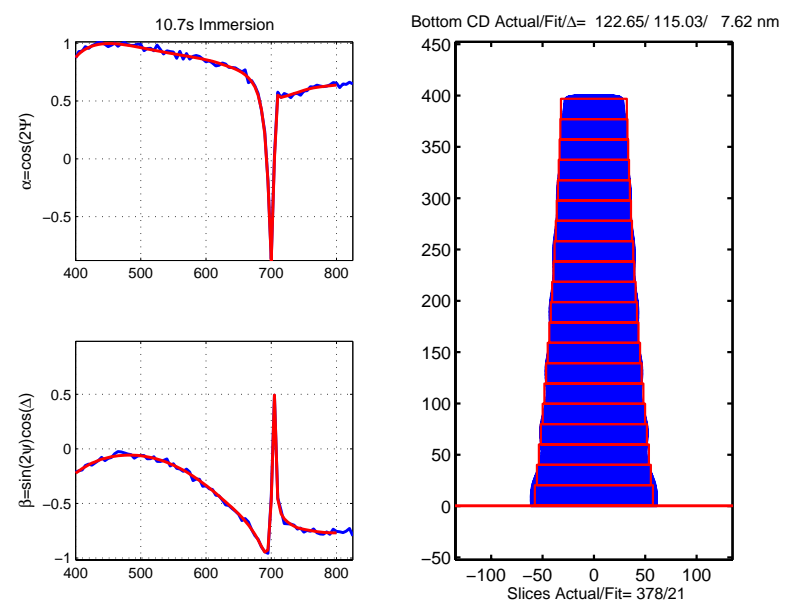

\begin{tabular}{|l|r|r|}
\hline Parameter & \multicolumn{1}{|l|}{ Value } & \multicolumn{1}{|c|}{$\begin{array}{c}68.3 \% \\
\text { Conf. } \\
\text { Limit }\end{array}$} \\
\hline $\begin{array}{l}\text { Seg 1 } \\
\text { Height (nm) }\end{array}$ & 396.171 & 2.066 \\
\hline $\begin{array}{l}\text { Seg 1 } \\
\text { Top Width (nm) }\end{array}$ & 63.450 & 4.644 \\
\hline $\begin{array}{l}\text { Seg 1 } \\
\text { Slope }\end{array}$ & 0.086 & 0.076 \\
\hline $\begin{array}{l}\text { Seg 1 } \\
\text { Curvature }\end{array}$ & 0.112 & 0.077 \\
\hline $\begin{array}{l}\text { Seg 2 } \\
\text { Height (nm) }\end{array}$ & 0.621 & 1.337 \\
\hline
\end{tabular}

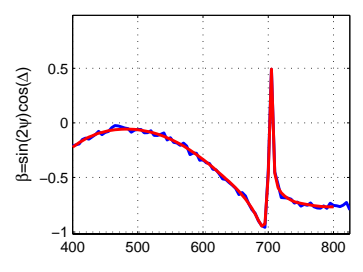

Figure 9 The data of Figure 8 is fit to the same curved-trapezoid/rectangle model but over a more limited wavelength range. The $\chi \therefore$ was $\sim 0.868$ for this fit.

\section{Instrumentation and Measurement Modes}

For all of the simulation cases reported above, we assumed specular reflection spectroscopic ellipsometry measurements were used. These could relatively easily be accomplished with windowed test cells as illustrated schematically below.

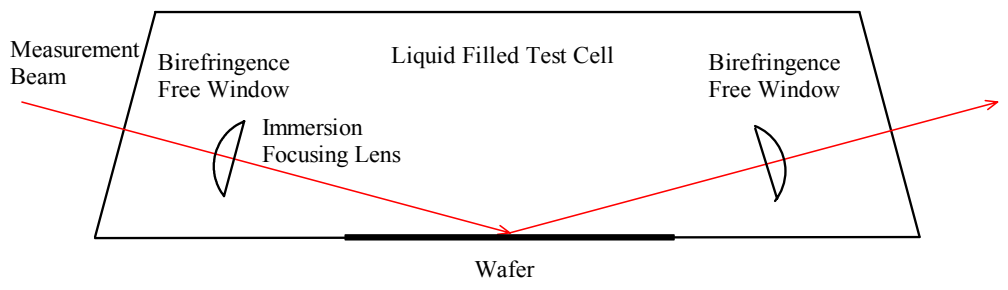

Figure 10 Schematic of immersion cell.

This same configuration could be used for spectroscopic reflectometry measurements. We also performed simulation investigations of parameter extraction experiments in the reflectometry mode. While spectroscopic ellipsometry is, in general, more sensitive and accurate, reflectometry measurements still yield very good accuracy in these applications and have the potential of higher speed at similar signal to noise ratios. For this application, the simultaneous measurement of both p- and s-mode reflectance using instrumentation such as that discussed in reference 5 would be particular useful. With modern, high speed CCD-cameras, this type of measurement could be made to be very fast.

We also numerically investigated the use of diffracted light information in a sort of darkfield approach. This is an immersion version of the previously proposed MOXIE approach. ${ }^{20}$ As is the case with darkfield microscopy, the scattered signal should be sensitive only to the pattern and not uniform films. For the cases simulated in this work, we found very good theoretical sensitivity for this measurement mode. Test cells with immersion lenses could be designed along the lines illustrated in Figure 11 and a useful range of scattered wavelengths could be collected. However, the magnitudes of the $-1^{\text {st }}$ order diffraction efficiencies for the photoresist grating structures in this study were very low (of order of a few percent at best). Addition of noise to this data to this data would have rendered it unusable for feature 
shape extraction. We believe these low diffraction efficiencies were the result of the relatively small diffractive index difference between the photoresist and the $\mathrm{H}_{2} \mathrm{O}$ medium. Thus, there is a trade-off in immersion mode scatterometry the reduction of the effective measurement wavelengths allows coupling to real diffracted modes for shorter period gratings, but the reduction of the refractive index difference between the line and the ambient (in the spacer region) reduces the effective scattering strength of the line. Of course, if the ambient refractive index exactly matches the index of the grating lines, there would be no scattering at all and the grating would effectively disappear to that measurement. This trick was exploited for thin film ellipsometry to investigate effects at $\mathrm{SiO}_{2}$-Si interfaces. ${ }^{21}$

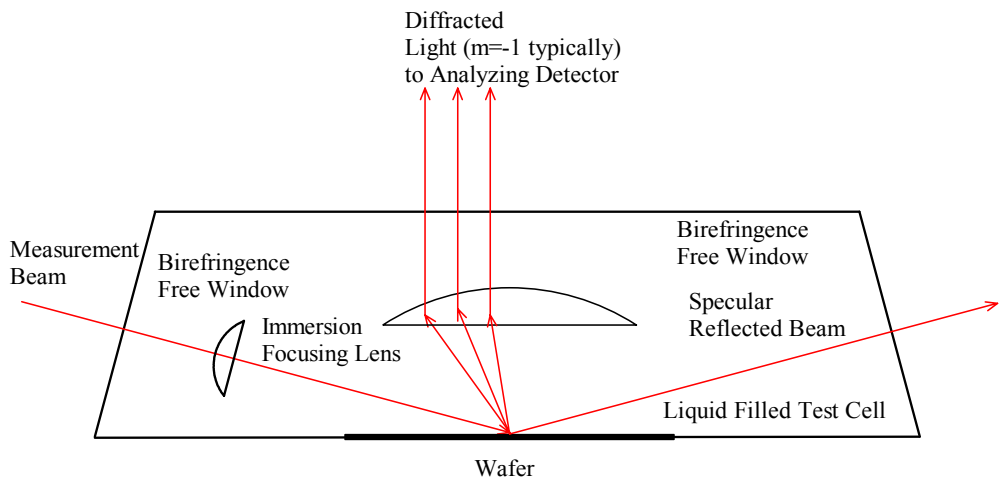

Figure 11 Schematic illustration of darkfield immersion measurements.

\section{Conclusions}

We have numerically investigated the use of immersion-mode scatterometry for nano-scale feature extraction. For very short period gratings, enhanced resolution of feature shapes should be offered by the immersion environment. Also, a significant secondary benefit of a smaller focused measurement spot size should be realized. For real-time resist profile measurements, the optical behavior of the developer may pose practical limitations, but preliminary simulations suggest that even a reduced measurement wavelength window will be useful for development model building. Existing spectroscopic ellipsometry and reflectometry hardware can be combined with immersion cells to implement this approach. Some hardware development would be required for the realization of high-speed immersion scatterometry measurement systems, but these efforts could borrow heavily from the work already done on immersion lithography steppers. Obviously, the stage requirements would not be as stringent as those of the steppers. Recent developments in high-speed CCD camera could enable very rapid data acquisition.

\section{References}

${ }^{1}$ M.E. Lee, C. Galarza, W. Kong. W. Sun, and F. L. Terry, Jr., "Analysis of reflectometry and ellipsometry data from patterned structures," Characterization and Metrology for ULSI Technology. 1998 International Conference. 23-27 March 1998; Gaithersburg, MD, USA, AIP-Conference-Proceedings. no.449; 1998; p.331-5.

${ }^{2}$ Niu Xinhui, N Jakatdar, Junwei-Bao, C.J. Spanos, “Specular spectroscopic scatterometry,” IEEE-Transactions-onSemiconductor-Manufacturing. vol.14, no.2; May 2001; p.97-111.

${ }^{3}$ J.Allgair and P. Herrera, "Spectroscopic optical metrology for process characterization and control," MicrolithographyWorld. vol.11, no.1; Feb. 2002; p.12, 14, 16, 23-4

${ }^{4}$ J. Opsal, H. Chu, Y. Wen, G. Li; “Fundamental solutions for real-time optical CD metrology,” SPIE Conference on Metrology, Inspection, and Process Control for Microlithography XVI, Santa Clara, CA, March , 4-7, 2002.

${ }^{5}$ B. S. Stutzman, H.-T. Huang, and F. L. Terry, Jr., "Two-channel spectroscopic reflectometry for in situ monitoring of blanket and patterned structures during reactive ion etching," J. Vac. Sci. Techn., B18, pp.2785-93 (2000). 
${ }^{6}$ Hsu-Ting Huang, Wei Kong, and Fred Lewis Terry, Jr., "Normal Incidence Spectroscopic Ellipsometry for Critical Dimension Monitoring,” Applied Physics Letters, vol.78, no.25; 18 June 2001; p.3983-5

${ }^{7}$ V. A. Ukraintsev, M. Kulkarni, C. Baum, K. Kirmse, Texas Instruments Inc.; M. Guevremont, S. Lakkapragada, K. N. Bhatia, P. P. Herrera, U. K. Whitney, "Spectral scatterometry for 2D trench metrology of low-k dual-damascene interconnect," SPIE Conference on Metrology, Inspection, and Process Control for Microlithography XVI, Santa Clara, CA, March 4-7, 2002.

${ }^{8}$ Kevin R. Lensing, Clint Miller, Geoff Chudleigh, Bryan Swain, Michael Laughery, Anita Viswanathan, "Scatterometry feasibility studies for 0.13-micron flash memory lithography applications: enabling integrated metrology," Proceedings of SPIE Volume 5375, pp. 307-16 (2004).

${ }^{9}$ Gowri P. Kota, Jorge Luque, Vahid Vahedi, Ashok Khathuria, Thaddeus G. Dziura and Ady Levy, “Advanced process control for poly-Si gate etching using integrated CD metrology,” Proc. SPIE 5378, pp. 105-115 (2004).

${ }^{10}$ Ivan Pollentier, Shaunee Y. Cheng, Bart Baudemprez, David Laidler, Youri van Dommelen, Rene Carpaij, Jackie Yu, Junichi Uchida, Anita Viswanathan, Doris Chin, Kelly Barry, Nickhil Jakatdar, "In-line lithography cluster monitoring and control using integrated scatterometry," Proc. SPIE 5044, pp. 90-6 (2003).

${ }^{11}$ Hsu-Ting Huang, Ji-Woong Lee, Brooke S. Stutzman, Pete Klimecky, Craig Garvin, Pramod P. Khargonekar, and Fred L. Terry, "Real Time In Situ Monitoring of Deep Sub- $\mu \mathrm{m}$ Topography Evolution during Reaction Ion Etching," Jr., SEMATECH AEC/APC Symposium, Lake Tahoe, NV., September 25-28, 2000.

${ }^{12}$ Hsu-Ting Huang, Ji-Woong Lee, Pete Klimecky, Pramod P. Khargonekar, and Fred L. Terry, Jr., "In Situ Monitoring Of Deep Sub- $\mu \mathrm{m}$ Topography Evolution And Endpoint Detection During Reactive Ion Etching," SEMATECH AEC/APC Symposium XIII, October 6-11, 2001, Banff, Alberta, Canada

${ }^{13}$ M.G. Moharam, Eric B. Grann, Drew A. Pommet, and T. K. Gaylord, "Formulation for stable and efficient implementation of the rigorous coupled-wave analysis of binary gratings," J. Opt. Soc. Am. A, vol. 12, pp 1068-76 (1995).

${ }^{14}$ Lifeng Li, "Use of Fourier series in the analysis of discontinuous periodic structures," J. Opt. Soc. Am. A, vol. 13, pp. 1870-6 (1996).

${ }^{15}$ Values taken from the Sorpa, SA database available on-line at www.sopra-sa.com

${ }^{16}$ Fred L. Terry, Jr., “Accuracy Limitations in Specular-Mode Optical Topography Extraction," Proc. SPIE 5038, pp. 574-558 (2003).

${ }^{17}$ William H. Press, Brian P. Flannery, Saul A. Teukolsky, and William T. Vetterling, Numerical Recipes: The Art of Scientific Computing, Cambridge Univ. Press, 1986, pp. 502ff.

${ }^{18}$ G.E. Jellison, "Spectroscopic ellipsometry data analysis: measured versus calculated quantities," Thin Sol. Films, vol. 313-314, pp. 33-9 (1998).

${ }^{19}$ www.eecs.umich.edu/ fredty/ (follow the links from this site).

${ }^{20}$ Christopher P. Ausschnitt, “A New Approach to Pattern Metrology,” Proc. SPIE 5375, pp. 51-65.

${ }^{21}$ C. Zhao, P.R. Lefebvre, and E.A.Irene, "A spectroscopic immersion ellipsometry study of $\mathrm{SiO}_{2}$-Si interface roughness for electron cyclotron resonance plasma and thermally oxidized Si surfaces," Thin Sol. Films, vol. 313-314, pp. $286-91$ (1998). 